\title{
DISTRIBUTION OPTIMIZATION BY MEANS OF BREAK QUANTITIES: A CASE STUDY
}

\author{
Roel Nass, Erasmus University Rotterdam \\ Rommert Dekker, Erasmus University Rotterdam \\ Willie van Sonderen-Huisman, Erasmus University Rotterdam \\ The Netherlands
}

In this paper we present a case study on physical distribution optimization in Western Europe. Finished goods of a similar nature can be delivered either from distribution centres (DC's) or form production service centres (PSC's). The delivery time via a DC is shorter, but higher costs are involved. The selection between the two ways of delivery is based on the stockmix and on the Break Quantities (BQ's). The set of products present at a DC is called the stockmix, which for efficiency reasons is restricted. If a product present at a DC is ordered and the order quantity is below the break quantity, the order is delivered from the DC and else form a PSC. The problem is to decide on the stockmix and the break quantity for each DC.

The objective is to minimize total distribution and handling costs subject to constraints on service. The distribution costs per kilogram from a certain PSC at a certain day depend on the total volume delivered from that PSC that day. This total volume depends on the BQ's of several DC's. Therefore, it was not possible to decompose the problem to determine a BQ for each DC separately. A further complication was that service constraints were formulated for the entire network, rather than for individual DC's. Our approach was as follows. A simulation program was built to determine total costs and service performance for given stockmixes and BQ combination using all actual orders received over a four-month period. We first considered three options for the stockmixes while using historical BQ's.

The best one was: stocking the products with most orders. Next the simulation program was run for a large set of $\mathrm{BQ}$ combinations. We fitted a simplified function to the total costs, to allow a nonlinear optimization by a standard NLP solver. Another simplified objective function was obtained by assuming $80 \%$ truck loads for deliveries from PSC's. The methods were compared with the best generated BQ combination on performance and computational effort. 